\title{
Tuberculum Sellae Meningiomas: Outcome of Twenty-eight Cases after Surgery
}

\author{
Rahman MA ${ }^{1}$, Jahan $N^{2}$, Bari MS², Mitra $\mathrm{PK}^{3}$, Hafiz $\mathrm{AH}^{3}$, Saha $\mathrm{AK}^{1}$, Mahmud $\mathrm{E}^{4}$
}

Conflict of interest: There is no conflict of interest relevant to this paper to disclose.

Funding Agency: was not funded by any institute or any group.

Contribution of Authors: Principal Investigator and Manuscript preparationData collection-

Scalp block with anaesthesia-

Editorial formatting-

Copyright: @2020bang.BJNS published by BSNS. This article is published under the creative commons CC-BY-NC license. This license permits use distribution (https://creativecommons. orgf/licences/by-nc/4-0/)reproduction in any medium, provided the original work is properly cited and is not used for commercial purposes.

Received: 02.02.20

Accepted: 06.04.20

\begin{abstract}
Objective: To present this series of surgically treated tuberculum sellae meningiomas with particular regard to visual compromises

Methods: A retrospective analysis was done on 28 patients (21 females) with meningiomas originating from the tuberculum sellae who underwent surgery between 2010 and 2019. The standard surgical approach of pterional craniotomy. Twelve meningiomas extended posteriorly onto the diaphragma sellae, 13 anteriorly to the planum sphenoidale, and 3 to the anterior clinoid process. 21 tumours involved the optic canal, one bilaterally. Follow up ranged from 6 to 12 months.
\end{abstract}

Results: Total microscopic resection was achieved in 28 patients. Median tumour size was $3.2 \mathrm{~cm}$. Postoperatively, visual acuity improved in 19 patients and deteriorated 3. Preoperative and postoperative visual acuity worsened with increasing duration of preoperative symptoms and with increasing age. Extension into the intraconal space was a negative predictor. Recurrence occurred in one cases. One patients died from causes unrelated to the tumour.

Conclusions: In the majority of patients with tuberculum sellae meningiomas, total resection may be achieved through a pterional approach with minimal complications.

Bang. J Neurosurgery 2020; 10(1): 39-44

\section{Introduction:}

Meningiomas are common extra-axial brain tumors that arise from the arachnoid cap cells. Suprasellar meningiomas account for about $10 \%$ of all meningiomas ${ }^{1}$. The two most common suprasellar locations are the tuberculum sella and planum sphenoidale, and they may extend to the diaphragm sella. The anatomic definition of the TS is the midline elevation anterior to the dorsum sella but behind the chiasmatic sulcus. This term has been used broadly to describe tumor originating from the TS, chiasmatic sulcus, limbus sphenoidale, and diaphragm sella and it grows in a subchiasmal position ${ }^{2}$. Tuberculum sellae meningiomas constitute $5-10 \%$ of intracranial meningiomas. Their location in the parasellar region, in close vicinity to hypothalamic structures and anterior vessels of the circle of Willis, TSMs are situated near important structures such as the pituitary stalk, major arteries, optic nerve and optic chiasm ${ }^{3,4,5}$. TSM displayed optic chiasm postero- superiorly and the optic nerves laterally, most commonly extend into both optic canals which causes visual impairment due to displacement of or adherence to the optic apparatus ${ }^{5}$, 6 . However the most common clinical presentation for Tuberculum sellae meningiomas are gradual visual compromise. The term "chiasmal syndrome," coined by Holmes and Sargeant in 1927, refers to a primary optic atrophy with a bitemporal field defect due to compression on optic pathway ${ }^{7}$. About 10 to $20 \%$ patients experiencing worsening of preoperative visual

1. Dr Md Atikur Rahman, Associate Professor, Neurosurgery Department, Bangabandhu Sheikh Mujib Medical University, Shahbag, Dhaka

Dr. Asit Kumar Saha, Associate Professor of Neurosurgery, Patuakhali Medical college,Patuakhali

2. Dr. Nwoshin Jahan, Resident, Neurosurgery Department, Bangabandhu Sheikh Mujib Medical University, Shahbag,

Dr. Mohammad Shahnawaz Bari, Resident \& Medical Officer, Neurosurgery Department, Bangabandhu Sheikh Mujib Medical University, Shahbag,

3. Dr. Pijush Kanti Mitra, Assistant Professor, Neurosurgery Department, Dhaka Medical College Hospital, Dhaka.

Dr. Ahsan Mohammad Hafiz, Assistant Professor, Neurosurgery Department, Uttara Adhunik Medical College Hospital, Dhaka.

4. Prof. Ehsan Mahmud, Ex-Professor, Neurosurgery Department, Dhaka Medical College Hospital, Dhaka

Address of Correspondence: Dr. Md. Atikur Rahman, Associate Professor, Neurosurgery Department, Bangabandhu Sheikh Mujib Medical University, Shahbag, Dhaka. Email: atiquessmc@yahoo.com, Mobile: +8801715011169 
function reported in many article 8 . However other less common symptoms and signs are headache, dizziness, seizures, endocrine disturbance, altered behavior, and cranial nerve deficits. The tumor may fill the sellar and suprasellar area, with dural attachment generally at the tuberculum sellae. As the tumor grows it can displace, stretch, or even encase vital structures. Vital structures and important anatomic landmarks bound the tuberculum sellae are laterally clinoid processes (fig:1), the internal carotid and the posterior communicating arteries; posteriorly the pituitary stalk, infundibulum, and the Lillequist membrane; superior boundary form by the optic chiasm, the lamina terminalis, and the anterior cerebral artery complex. Which make the space is quite small, (mean length $8 \mathrm{~mm}$, mean width $11 \mathrm{~mm})^{9}$. Compromise of the optic apparatus is an important feature for decision making ${ }^{9,10}$. The aim of surgery is to relieve the mass effect upon the optic apparatus. Several surgical approaches such as subfrontal, bifrontal, unilateral frontal, supraorbital keyhole, pterional have been proposed for the resection of TSM, and more recently in different article neurosurgical endoscopic techniques have been introduced as an option during removal of skull base tumors both transcranially and endonasally 9,11 .

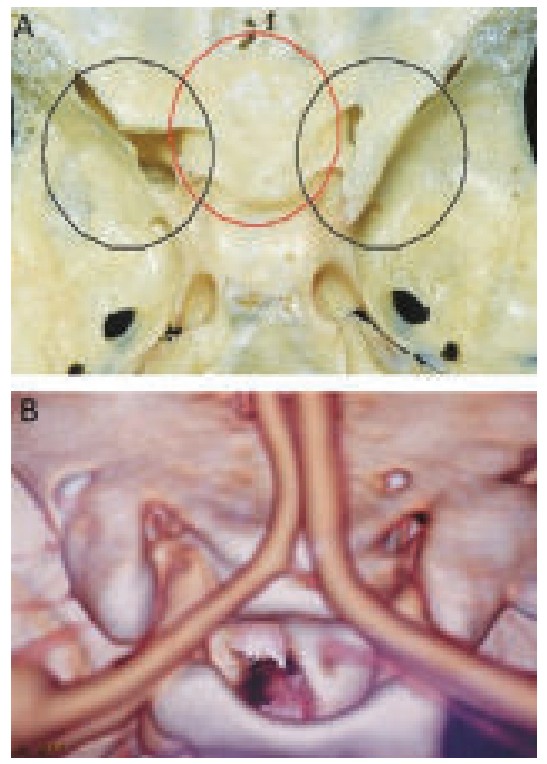

Fig.-1: Anatomy of tuberculum sellae. A - The region of the tubercullum sellae com-prises the space belonging to the sphenoid bone between the two anterior clinoids (red circle), limited posteriorly by the dorsum sellae, before the planum sphenoidale. The optical column and the optic canal on each side form the posterior lateral limit (black circles). B - CT angiography with 3D reconstruction shows the relationship between the arteries and the tuberculum sellae.

\section{Methods:}

Patient characteristics the series consisted of 28 patients with tuberculum sellae meningiomas, all of whom underwent surgery from December 2010 to December 2019. The cohort comprised 21 women and 7 men (female to male ratio, 3:1) (Fig 2). Follow up data were available from 3 to 12 months, with a median of 7 months. The mean age of the patients was 36 years (range 24 to 62). Ophthalmological examination consisted of testing the patients' visual acuity (Snellen notation), fundoscopy, and Goldmann perimetry for visual field defects. A change of $>2$ lines indicated improvement or deterioration. Oculomotor function was evaluated, along with other neurological functions. Endocrinological tests (adrenal, thyroid, and gonadal axes, specific gravity of the urine, fluid balance) were done preoperatively and one week and three months postoperatively. All patients underwent evaluation by computed tomography (CT) or magnetic resonance imaging (MRI) with and without contrast agent. High resolution CT showed hyperostosis of the planum sphenoidale and tuberculum sellae or calcification within the tumour. Both T1 and T2 weighted MRI were done in three planes to analyses the relation to vascular and neighbouring structures. Follow up MRI was scheduled at three and 12 months postoperatively. Information on clinical history, signs, surgical approach, and outcome was obtained retrospectively by review of the patient's case notes and the radiological reports. All 28 meningiomas originated from the tuberculum sellae. Twelve extended posteriorly into the diaphragmal region, 13 anteriorly to the planum sphenoidale, and 3 to the anterior clinoid process. 21 involved the optic canal (fig 1) and one bilaterally. Twelve extended along the optic canal into the intraconal orbital compartment. One presented as a large bulbiform orbital mass; this patient presented with one large mass at the tuberculum sellae

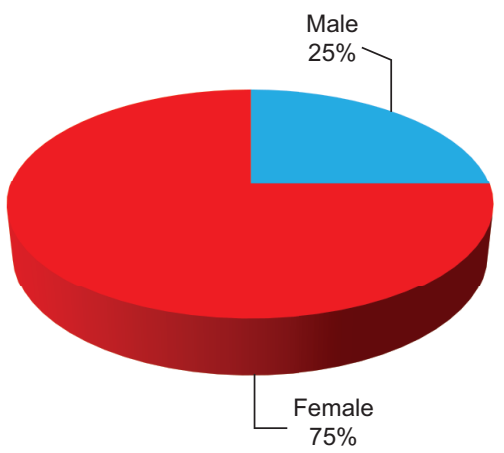

Fig.-2: Male and Female distribution 
and the second mass around the optic nerve. It is probable that this intraorbital tumour had extended into the intracranial space, forming a second tumour nodule. The size of the tumour varied from $3 \mathrm{~cm}$ to $5 \mathrm{~cm}$.

\section{Surgical technique:}

All operations were carried out through frontotemporal approach, on the side of visual deterioration. In bilateral involvement the right side was preferred. This pterional approach is well described. ${ }^{8} 9$ Drainage of cerebrospinal fluid (CSF) was done by lumbar drainage and later by opening the basal cisterns. The sylvian fissure was opened in specific cases and the M1 segment of the middle cerebral artery exposed. The internal carotid artery was identified and this led to the ipsilateral optic nerve, which might be covered by tumour. The anterior tumour capsule was opened and the basal blood supply was interrupted by lifting the tumour and coagulating the feeding arteries until the ipsilateral optic nerve appeared. The tumour was further debulked to reduce its volume until the contralateral optic nerve became visible. The tumour located medial of the ipsilateral optic nerve was then removed from the skull base. This step was followed by dissection of the arachnoidal plane from the ipsilateral optic nerve. After interruption of the basal blood supply, the tumour became soft and anaemic and thus could easily be detached from the arachnoid of the gyrus rectus, the A1 segment of the anterior cerebral artery, or the anterior communicating artery. Finally, the tumour was removed from the $A 1$ segment and the chiasm. Protection of the optic and chiasmatic blood supply is extremely important to preserve vision. In particular, the small vessels from the carotid artery to the optic nerve have to remain in their arachnoidal layer and should not be occluded. In cases of hyperostotic planum sphenoidale the basal dura was excised and the bone was drilled away. The defect was covered with fat, freeze dried dura, and fibrin glue. In optic canal involvement the first $3-5 \mathrm{~mm}$ of the optic canal, which are fibrous, were opened. The bony roof was drilled away if there was further tumour extension. The ophthalmic artery below the optic nerve was exposed and preserved. If necessary, the optic nerve sheath was opened until the annulus of Zinn was reached and the tumour around the optic nerve was carefully removed. In contrast to optic nerve sheath meningiomas, tumour mass in the optic canal in tuberculum sellae meningiomas could easily be dissected from the optic nerve. The technique has only slightly changed over the years. The most important steps remain the interruption of the basal blood supply and hollowing of the tumour. The most important structure to be exposed is the contralateral optic nerve to obtain an anatomical overview. Close to the optic nerves, no active coagulation was used and the tumour was sucked within its arachnoidal plane. In recurrent tumour growth no radical resection was done. Deroofing of the optic canal was carried out whenever intracanalicular tumour growth was suspected. The tumour is always located in the basal part of the canal. With deroofing of the canal, the optic nerve can be mobilised and then the tumour below the nerve can be sucked away. This procedure prevents recurrent tumour growth.

\section{Results:}

Signs and symptoms

The mean duration of symptoms was 24 months (range 6 to 46). The preoperative duration of symptoms correlated negatively with preoperative visual acuity. Postoperative recovery of visual deficits was worse within the group of patients with deficits lasting longer than six months. There was a clear correlation between age and preoperative visual disturbances. Visual impairment was the initial symptom in 25 patients, chiasmal compression resulted in central scotoma and temporal anopia in one eye and incomplete temporal anopia in the other eye. Compression of the chiasm could provoke the classical bitemporal hemianopia with reduction in central visual acuity in one or both eyes. Involvement of the intracranial optic nerve at the junction to the chiasm led to the anterior junction syndrome with ipsilateral central scotomas and contralateral temporal anopia. Lesions of the optic tract resulted in incongruent homonymous hemianopia in eleven patients. Three patients had irregular small unclassified visual field defects and four were blind in one eye. Hyposmia was present in five patients. Tumour related endocrinological abnormalities were seen in three patients.

\section{General history}

One patients suffered from multiple intracranial or spinal meningiomas, and three from optic nerve sheath meningiomas. Four patients had undergone operation for tuberculum sellae meningiomas in other neurosurgical departments and were referred to our centre for reoperation. One patient was misdiagnosed as a pituitary adenoma and first operated on by a trans-sphenoidal approach. 


\section{Surgery}

The standard approach was the intradural pterional approach. Twelve patients were operated on through a right sided approach and Ten patients through bifrontal approach approach. Tumour size influenced the preservation of the arachnoid. With respect to preoperative and postoperative visual acuity, there was no difference between the groups with preserved and non-preserved arachnoid. However, there was a tendency to worse postoperative visual acuity in the group with non-preserved arachnoid medial to the optic nerve. The pituitary stalk could be preserved in all cases. Total microscopic resection was achieved in 28 patients. Three patients had subtotal resection owing to small tumour rests around the vessels or the optic nerve or to bone involvement. The extent of resection depends on tumour size. The ipsilateral optic canal was explored in sixteen patients. The dural part was involved in 22 cases. The bony roof was drilled away in all patients who had further tumour extension. Bilateral involvement of the optic canal was seen in nine patients. However, the extent to which the optic canal was opened did not influence postoperative visual acuity. Only tumour size was associated with the extent of canal involvement. However, only deroofing of the optic canal allows total tumour removal below the optic nerve within the optic canal. Neither the extent of resection nor the size of the tumour influenced postoperative visual acuity.

\section{Complications}

One patients (60 years) died one month postoperatively. Two patient had to undergo a second operation for residual. One patients had a temporary cerebrospinal fluid leak and were adequately treated by lumbar drainage and one suffered from an epileptic seizure in the first postoperative week. One patient received hydrocortisone as replacement therapy for six months. One patients developed permanent hyposmia and one developed anosmia.

\section{Outcome:}

On discharge, the absolute postoperative visual acuity improved in 19 patients, remained unchanged in 5 , and deteriorated in three. Preoperative vision correlated highly with postoperative vision. Age was negatively correlated with the postoperative visual acuity. Longer duration of preoperative symptoms correlated with worse recovery. Deterioration of vision was not associated with involvement of the optic canal. In these cases, there was a tendency to interruption of the arachnoidal plane. Thus deterioration of visual acuity seems to be a vascular problem. Nineteen patients with visual field defects improved markedly postoperatively. In ten patients the defects resolved completely. However, seven patients worsened. Two patient developed diabetes insipidus.

\section{Follow up}

Vision generally improved rapidly within the first weeks after surgery, but continued to improve for the next year. At follow up, the absolute visual acuity improved further in two patients and worsened in one. Only one patient with bad vision improved further. Patients older than 50 years had worse recovery. Vision did not deteriorate with longer follow up.

\section{Recurrence}

Recurrence was two in two cases 23 and 36 months postoperatively. However, follow up varied from 6 to 12 months. One of these patients became symptomatic with a new visual field defect and the other with headache. The case with early recurrence had a history of multiple meningiomas and the $4 \mathrm{~cm}$ tumour was only subtotally resected at the first operation. Both patients underwent reoperation with good results.

\section{Discussion :}

Tuberculum sellae meningiomas are relatively common, The neurological, visual, and long-term outcomes are determined by the success of the surgical removal of tumor. The extent of the surgical resection of the tumor will determine the tumor recurrence and regrowth. Peritumoral edema and tumor calcification are the indicator of firm nature of the tumor. The ability of meningiomas to invade the parenchyma can also be related to the peritumoral edema, and it can influence the risk of recurrence. The extent of ventriculomegaly and surrounding edema suggested a relationship to and intimacy with the surrounding brain and adjoining structures. Angiography is helpful in exactly defining the feeding vessels and the extent of tumor vascularity and in suggesting the feasibility of embolization. Different author in various article has been recommended that the optic canal should be opened in order to remove small pieces of residual tumor as residual tumors in the optic canal can also be a source of tumor recurrence $5,12,13,14$. Different surgical routes can be used for Tuberculum Sellae Meningiomas; it allows the neurosurgeon to make decisions concerning route preference, tactics and strategies. To correctly select 
the surgical approach, it is necessary to understand the advantages and disadvantages of each procedure and some factors must be considered by the neurosurgeon in making the right decision. To correctly select the surgical approach, it is necessary to understand the advantages and disadvantages of each procedure and some factors must be considered by the neurosurgeon in making the right decision? . According to Goel et al. Younger patients tolerated the surgical procedure better than older patients. Systemic factors, such as hypertension and diabetes mellitus, could affect the surgical procedure and outcome to a certain degree. The extent of visual deficit is the single most important factor that determined the course of surgery. The more extensive the involvement of the optic nerve as depicted by the extent of visual deficit, the more intense the relationship of the optic nerve and the internal carotid artery and its branches to the tumor, and consequently, the more difficult the dissection ${ }^{14}$. The most important factor during management of TSM should be give special attention are: (a) the anatomical characteristics of the tumor, such as size, consistency and its relationship with neurovascular areas (optic device, ICASs, cavernous sinuses, pituitary, among others); (b) patient condition and symptoms presented; (c) the experience of the neurosurgeon, a decisive factor in the choice of the approach. The aim of surgery is to achieve improvement of vision. So the key to preserving visual function is to minimize direct manipulation or trauma to the optic nerves and avoid injury to the blood supply of the optic apparatus9.Various authors have considered the size of the tumor to be a crucial factor that indicates possible surgical difficulties, moreover during pregnancy the vascularity of the tumor is more and extensive perilesional edema leading to surgical difficulties. Large tumors cause more severe stretching of the adjoining nerves and vessels, and consequently, the resection is more difficult ${ }^{14}$. To avoid such type of difficulties the initial debulking of the tumor should start from its center, where no vital structures are present. The arachnoid plane is then delineated, starting at the contralateral optic nerve and working toward the undersurface of the optic chiasma and then along the ipsilateral nerve. However during resection of the meningioma, small vessels observed in the stretched arachnoid layer should not be coagulated. By preserving these vessels, there is a better chance for visual function improvement ${ }^{9}$. According to Gadget et al. in cases of TSM with a significant level of optic nerve compromise and with limited lateral extension, endoscopic endonasal resection is an excellent option but if there are medial optic canal invasion transcranial surgery is must 9,14 .

\section{Conclusion :}

The most common clinical presentation of a TSM is visual deterioration and the main aim of surgery is complete tumor excision with maintaining or improvement of visual function. Tuberculum sellae meningiomas should be resected early in their course in patients presenting with symptoms of optic chiasm compression. Adequate preoperative visual field assessment is necessary to allow for objective evaluation of postoperative visual results. The prognosis and visual outcome are excellent in patients with short preoperative visual affection; intact arachnoid membrane; absence of arterial encasement and peritumoral edema.

\section{Referrence:}

1. Al-Mefty $\mathrm{O}$, et al. Microsurgical removal of suprasellar meningiomas. Neurosurgery. 1985;16(3):364-72.

2. Sekhar LN, Hadjipanayis CG, Recinos PF. 17 Planum/ Tuberculum Sella Meningiomas. InEndoscopic and Keyhole Cranial Base Surgery 2019 (pp. 223-249). Springer, Cham.

3. Wilk A, G ZI WP, Koziarski A. Outcome assessment after surgical treatment of tuberculum sellae meningiomas-a preliminary report. Turk Neurosurg. 2016 Nov 1;26:824-32.

4. Rohringer M, Sutherland GR, Louw DF, Sima AA. Incidence and clinicopathological features of meningioma. Journal of neurosurgery. 1989 Nov 1;71(5):665-72.

5. Bhattarai R, CaoFeng L, Ying G. Microsurgical Management of Tuberculum Sellae Meningiomas by the supraorbital keyhole eyebrow approach: Surgical Outcome. Nepal Journal of Neuroscience. 2017 Dec $1 ; 14(3): 26-32$.

6. Chi JH, McDermott MW. Tuberculum sellae meningiomas. Neurosurgical focus. 2003 Jun 1;14(6):1-6.

7. Khallaf M. Tuberculum Sellae Meningiomas: Nuances in Treatment. Open Journal of Modern Neurosurgery. 2019 May 8;9(3):227-36.

8. Jallo GI, Benjamin V. Tuberculum sellae meningiomas: microsurgical anatomy and surgical technique. Neurosurgery. 2002 Dec 1;51(6):1432-40.

9. Estevão IA, Camporeze B, Matricardi G, Silva Júnior PD, Gripp DA, Santiago NM, Aguiar PH. Tuberculum sellae meningioma: Is there an ideal approach?. MedicalExpress. 2017 Aug;4(4).

10. Perondi GE, Isolan GR, de Aguiar PH, Stefani MA, Falcetta EF. Endoscopic anatomy of sellar region. Pituitary. 2013 Jun 1;16(2):251-9. 
11. Chen G, Wang Z, Zhou D. Lateral supraorbital approach applied to sellar tumors in 23 consecutive patients: the Suzhou experience from China. World journal of surgical oncology. 2013 Dec 1;11(1):41.

12. Bassiouni H, Asgari S, Stolke D. Tuberculum sellae meningiomas: functional outcome in a consecutive series treated microsurgically. Surgical neurology. $2006 \mathrm{Jul}$ 1;66(1):37-44

13. Otani N, Muroi C, Yano H, Khan N, Pangalu A, Yonekawa $\mathrm{Y}$. Surgical management of tuberculum sellae meningioma: role of selective extradural anterior clinoidectomy. British journal of neurosurgery. 2006 Jan 1;20(3):129-38.

14. Goel A, Muzumdar D, Desai KI. Tuberculum sellae meningioma: a report on management on the basis of a surgical experience with 70 patients. Neurosurgery. 2002 Dec 1;51(6):1358-64.

15. Gadgil N, Thomas JG, Takashima M, Yoshor D. Endoscopic resection of tuberculum sellae meningiomas. Journal of Neurological Surgery Part B: Skull Base. 2013 Aug;74(04):201-10. 